Research Article

\title{
Serum Soluble ST2 and Diastolic Dysfunction in Hypertensive Patients
}

\author{
Anca Daniela Farcaș, ${ }^{1,2}$ Florin Petru Anton, ${ }^{1,2}$ Cerasela Mihaela Goidescu,, ${ }^{1,2}$ \\ Iulia Laura Gavrilă, ${ }^{2}$ Luminiţa Animarie Vida-Simiti, ${ }^{1,2}$ and Mirela Anca Stoia ${ }^{1,2}$ \\ 1 "Iuliu Hatieganu" University of Medicine and Pharmacy, Cluj-Napoca, Romania \\ ${ }^{2}$ Emergency Clinical County Hospital, Cluj-Napoca, Romania \\ Correspondence should be addressed to Florin Petru Anton; florinantonfr@yahoo.com
}

Received 30 January 2017; Revised 23 March 2017; Accepted 29 March 2017; Published 8 May 2017

Academic Editor: Massimiliano M. Corsi Romanelli

Copyright (c) 2017 Anca Daniela Farcaş et al. This is an open access article distributed under the Creative Commons Attribution License, which permits unrestricted use, distribution, and reproduction in any medium, provided the original work is properly cited.

\begin{abstract}
Background. Echocardiographic evaluation of left ventricular (LV) structural and functional alterations in hypertension has some limitations, potentially overcome by using biomarkers. ST2, a prognostic biomarker for heart failure and myocardial infarction patients, was less studied in hypertension. Aim. To analyze the relationship between serum ST2 levels and diastolic dysfunction (DD) in hypertension. Method. We enrolled 88 hypertensive outpatients (average age 65 years, $69.3 \%$ females) in a prospective study, stratified for presence of LV hypertrophy (LVH). For each patient clinical examination, lab workup (routine and serum ST2 levels) and echocardiography were performed. Results. Hypertensive patients with LVH had higher age, pulse pressure, mean arterial pressure, and serum ST2, while having lower serum albumin than those without LVH. Serum ST2 levels correlate with parameters of LV remodeling and DD. We found that $5.3 \%$ of ST2 level variability was caused by a 1 -unit variation of cardiovascular risk. We identified cut-off values for discriminating hypertension with LVH versus that without LVH and grade 2 DD versus normal diastolic performance. Conclusion. ST2 could be used as diagnostic biomarker for cardiac remodeling and altered diastolic performance in hypertension, providing additional data to echocardiography. It could represent a milestone in early detection of cardiac performance alteration.
\end{abstract}

\section{Introduction}

Hypertension is a major cardiovascular (CV) risk factor associated with a high morbidity and mortality rate as a result of the structural alterations and functional imbalance $[1,2]$. Left ventricular (LV) hypertrophy (LVH) caused by pressure overload is a prognostic marker associated with a 3-fold increase in the risk for acute myocardial infarction (AMI) or death [3] and leads to heart failure (HF) [1]. Structural alterations specific to LVH may result in diastolic failure and are associated with impaired relaxation and increased LV filling pressure [4]. In contrast, concentric remodeling-that is, associated with impaired LV relaxation-does not appear to predict high filling pressure [5].
Echocardiography is a noninvasive method for identification and assessment of cardiac remodeling and its functional outcome-the diastolic dysfunction (DD) [6]. Several parameters assessed by echocardiography have a prognostic value for $\mathrm{HF}$ and $\mathrm{CV}$ death $[6,7]$. Although feasible, with rates ranging from $93 \%$ for the Valsalva manoeuver to over $99 \%$ for mitral flow parameters and tissue Doppler, ambulatory echocardiographic evaluation of LV diastolic dysfunction still fails to accurately quantify it in $6-19 \%$ of cases $[7,8]$. Documenting the changes in diastolic performance, especially the transition from grade 1 to grade 2 , in all patients with CV disease and hypertension is difficult [6]. This is where algorithms for echocardiographic diagnosis of LVDD come into play, including state-of-the-art technology and parameters with proven diagnostic and prognostic ability. 
Therefore, it is of paramount importance to identify biomarkers able to detect early stages of disease and/or monitor the progression, especially in the settings where cardiac imaging is hindered by specific comorbidities (such as obesity and lung diseases), lack of accessibility, high cost, or inexperienced operators. Such biomarkers could also be used in conjunction with other biomarkers $[9,10]$ or available imaging methods, thus providing additional information.

Studies have shown that ST2 is a useful biomarker to predict the evolution in both patients with [11] and without manifest CV disease but with CV risk factors (CVRF) [12, 13]. ST2 is a protein with two isoformes-a membrane-bound receptor form (ST2L) and a soluble form (sST2) secreted by cardiac myocytes exposed to stretch [14]. Interleukin-33 (IL-33) has recently been identified as the functional ligand of ST2L. Their interaction is required for the activation of the cellular cascade of events that protect the myocardium from hypertrophy and fibrosis $[14,15]$ by opposing the effects of angiotensin-II on myocytes. High levels of sST2 have a detrimental effect due to its action as a decoyreceptor by IL-33 neutralization, thus limiting its availability to bind to ST2L. Although in the general population, it has been shown that sST2 levels are associated with systolic blood pressure and HBP treatment [13], only few studies evaluating the correlations between ST2 levels, cardiac structural alterations, and functional changes in hypertensive patients are available.

The objectives of our study were to evaluate the correlation between ST2 levels and LVH and to assess the role of ST2 for the diagnosis of LVDD in hypertensive patients.

\section{Patients and Methods}

2.1. Study Group. This is an observational prospective study in which 88 hypertensive patients were enrolled from the outpatient clinic of the Cardiology Department of the Emergency Clinical County Hospital in Cluj-Napoca. Hypertension was diagnosed according to the ESH/ESC guidelines [16] - systolic blood pressure (SBP) $\geq 140 \mathrm{mmHg}$ and/or diastolic $(\mathrm{DBP}) \geq 90 \mathrm{mmHg}$. Exclusion criteria included the following: other heart diseases (ischemic HF, congenital $\mathrm{HF}$, valvular $\mathrm{HF}$, myocarditis, acute or chronic $\mathrm{HF}$, and acute coronary syndromes), chronic inflammatory and acute infectious diseases, and pulmonary diseases (COPD, asthma, and sleep apnea). Based on LVH as assessed by echocardiography, patients were assigned to group A ( $n=39$, LVH absent) and group B ( $n=49, \mathrm{LVH}$ present). Here, we present the baseline data pertaining to the objectives of the analysis included in this manuscript. All patients signed an informed consent at enrollment before any study procedures. The study was approved by the local Ethics Committee.

2.2. Study Protocol. Demographic data, risk factors, and previous medical history were collected from patients' files, interview, and clinical examination, including blood pressure, weight, and height measurements. We used SBP and DBP measurements to calculate pulse pressure (PP, difference between the SBP and DBP values) and mean arterial pressure (MAP, arithmetical mean of the SBP and DBP values). Body mass index (BMI) was calculated as weight $(\mathrm{kg}) /[\text { height }(\mathrm{m})]^{2}$. Patients with a BMI below $24.99 \mathrm{~kg} / \mathrm{m}^{2}$ were considered having normal weight; those with a BMI between $25 \mathrm{~kg} / \mathrm{m}^{2}$ and $29.99 \mathrm{~kg} / \mathrm{m}^{2}$, having overweight; and those with a BMI over $30 \mathrm{~kg} / \mathrm{m}^{2}$, being with obesity. Additional CV risk was evaluated according to the ESC guidelines on hypertension [16].

Blood samples were collected, and cardiac echocardiography was performed in all enrolled patients at baseline. Blood samples for the evaluation of total, LDL, and HDL cholesterol; triglycerides; glucose; creatinine; serum albumin; uric acid; natrium; potassium; sST2 and NTproBNP were collected in the morning, after overnight fasting and after a period of 5-10-minute rest. Biochemical measurements were performed in the day of collection using routine enzymatic methods (Konelab 30, Thermo Fisher Scientific Inc., Finland). Samples for sST2 and NTproBNP were centrifuged for 15 minutes at $1000 \times \mathrm{g}$; serum was stored at $-20^{\circ} \mathrm{C}$ until measurement. sST2 was assessed using a Soluble ST2/ILl1R4 (human) ELISA kit (Aviscera Bioscience Inc., Santa Clara, CA 95051, USA) and an ELISA Tecan Sunrise reader. For the ST2 assay, the analytical limit of detection (sensitivity) was $5 \mathrm{pg} / \mathrm{mL}$; intra-assay coefficients of variation (\%) were $4-6 \%$ and interassay coefficients of variation (\%) were $8-10 \%$.

The plasmatic levels of NTproBNP were determined using the ELISA method (Biomedica $\mathrm{GmbH} \mathrm{Au}$, detection limit $5 \mathrm{pmol} / \mathrm{L}$, CV intra-assay 4\%, CV interassay 3.8\%).

The echocardiography was performed using a $2-5 \mathrm{MHz}$ probe on a Siemens Acuson X300 ultrasound machine by an experienced echocardiographer who was unaware of the medical history of the patients, in compliance with the recommendations of the American Society of Echocardiography and the European Association of Cardiovascular Imaging $[17,18]$, with a focus on cardiac remodeling and systolic and diastolic function. Systolic and diastolic measurements of the cardiac walls and chambers were obtained in M mode. LV mass was computed using the modified Devereux formula [6]; values $>95 \mathrm{~g} / \mathrm{m}^{2}$ in women and $>115 \mathrm{~g} / \mathrm{m}^{2}$ in men were considered suggestive for the LVH diagnosis. LV ejection fraction (LVEF) was estimated using Simpson's biplane method. Diastolic performance was evaluated using the mitral diastolic flow pattern parameters ( $E$ and $A$ waves [early and late diastolic peak filling velocities]), $E / A$ waves' ratio, mitral $E$ wave deceleration time (EDT), isovolumetric relaxation time (IVRT) [6], and tissue Doppler at the mitral annulus (septal and lateral). e'm (peak early diastolic mitral annulus velocity by tissue Doppler), E/e'm, left atrium area and volume (indexed to the body surface area) values were computed in a 4 -chamber apical view. The myocardial performance index was computed as (IVCT + IVRT)/LVET (ICVT $=$ isovolumetric contraction time, IVRT $=$ isovolumetric relaxation time, and LVET = ejection time). Diastolic dysfunction was defined according to the American Society of Echocardiography and the European Association of Cardiovascular Imaging recommendations [18, 19] as follows: grade I (abnormal relaxation pattern) $-E / A<0.8$, deceleration time $(\mathrm{DT})>220 \mathrm{~ms}$, IVRT $\geq 100 \mathrm{~ms}$, septal e' $<8 \mathrm{~cm} / \mathrm{s}$, and $E / \mathrm{e}^{\prime} \mathrm{m} \leq 8$; grade II (pseudonormal filling) - DT between 160 and $220 \mathrm{~ms}, E / A$ between 0.8 and 1.5 , septal $\mathrm{e}^{\prime}<8 \mathrm{~cm} / \mathrm{s}$, 
TABLE 1: Clinical and demographic data for the study group.

\begin{tabular}{|c|c|c|c|}
\hline & & Patients' number (\%) & $p$ \\
\hline \multirow{2}{*}{ Gender } & Men & $27(30.7 \%)$ & \\
\hline & Women & $61(69.3 \%)$ & 0.000 \\
\hline \multirow{2}{*}{ Location } & Urban & $51(59.3 \%)$ & \\
\hline & Rural & $35(40.7 \%)$ & .054 \\
\hline \multirow{3}{*}{ Hypertension } & Stage 1 & $16(18.28 \%)$ & $.002^{\mathrm{a}}$ \\
\hline & Stage 2 & $42(47.72 \%)$ & $.03^{\mathrm{b}}$ \\
\hline & Stage 3 & $30(34 \%)$ & $.000^{c}$ \\
\hline \multirow{3}{*}{ Additional CV risk } & Very high & $33(38.4 \%)$ & $.04^{\mathrm{d}}$ \\
\hline & High & $38(44.2 \%)$ & $.000^{\mathrm{e}}$ \\
\hline & Moderate & $15(17.4 \%)$ & $.002^{\mathrm{f}}$ \\
\hline \multirow{2}{*}{ Controlled hypertension } & No & $30(34.5 \%)$ & \\
\hline & Yes & $57(65.5 \%)$ & $.000^{\mathrm{g}}$ \\
\hline \multirow{4}{*}{ Dyslipidemia } & Without & $8(9.3 \%)$ & \\
\hline & Hypercholesterolemia & $46(53.5 \%)$ & \\
\hline & Mixed & $27(31.4 \%)$ & \\
\hline & Hypertriglyceridemia & $5(5.8 \%)$ & \\
\hline \multirow{2}{*}{ Smoking } & No & $67(77.9 \%)$ & \\
\hline & Yes & $19(22.1 \%)$ & $.001^{\mathrm{g}}$ \\
\hline \multirow{3}{*}{ Weight status } & Normal & $34(39.5 \%)$ & $.04^{\mathrm{h}}$ \\
\hline & Overweight & $31(27 \%)$ & $.45^{\mathrm{i}}$ \\
\hline & Obese & $21(24.4 \%)$ & $.03^{\mathrm{j}}$ \\
\hline \multirow{2}{*}{ Diabetes } & Absent & $56(65.1 \%)$ & \\
\hline & Present & $30(34.9 \%)$ & $.001^{\mathrm{k}}$ \\
\hline
\end{tabular}

${ }^{a}$ Stage 1 versus stage 2 ; ${ }^{b}$ stage 2 versus stage 3 ; ${ }^{c}$ stage 1 versus stage 3 ; ${ }^{d}$ very high versus high; ${ }^{e}$ high versus moderate; ${ }^{f}$ very high versus moderate; ${ }^{g}$ no versus yes;

${ }^{h}$ normal versus overweight; ${ }^{\mathrm{i}}$ overweight versus obese; ${ }^{\mathrm{j}}$ normal versus obese; ${ }^{\mathrm{k}}$ present versus absent. CV: cardiovascular.

and E/e'm between 9 and 12; and grade III (restrictive filling) $-E / \mathrm{A} \geq 2$, DT $<160 \mathrm{~ms}$, IVRT $\leq 60 \mathrm{~ms}, E / \mathrm{e}^{\prime} \mathrm{m} \geq 13$ (or E/septal e' $\geq 15$ and E/lateral e' $>12$ ).

\section{Statistical Analysis}

Statistical analysis was performed with SPSS 16. Group comparison was done using the chi-square test for categorical variables, Student test for continuous variables with normal distribution, and Mann-Whitney $U$ test for continuous variables with abnormal distribution and ordinal variables. Correlation coefficients were calculated by linear regression analysis, and multiple regression analysis was applied for the analysis of the dependency between variables. Because sST2 did not have a normal distribution, we calculated its log-transformed values which were used as a dependent variable in the regression analyses. The performance of various cutoffs of sST2 for predicting the presence of LVH in patients with arterial hypertension and grade 2 diastolic dysfunction was evaluated using area under the receiver operating curve (AUC) and calculating the sensitivity and specificity. We also calculated AUC to compare the performance of sST2 in predicting the presence of grade 2 diastolic dysfunction with the one of proBNP, currently accepted as a gold standard for the assessment of ventricular dysfunction. A value of $p<0.05$ was considered statistically significant.

\section{Results}

The study group included 88 hypertensive patients with a mean age of 65 years (range 19 years to 85 years), of which $69.3 \%$ were female. The baseline demographic characteristics are presented in Table $1.47 .72 \%$ of patients had stage 2 hypertension and $34 \%$ had stage 3 hypertension. BP was controlled in $57 \%$ of patients. Several CV risk factors (modifiable or not) were present in the study group. $90.7 \%$ of the patients had dyslipidemia: 53.5\% hypercholesterolemia, $5.8 \%$ hypertriglyceridemia, and $31.4 \%$ mixed dyslipidemia. $22.1 \%$ were smokers and $51.4 \%$ had increased body mass index ( $27 \%$ were overweight and $24.4 \%$ obese). Diabetes was found in $34.9 \%$ of patients.

Table 2 summarizes the clinical and laboratory data of the two study groups. Patients with LVH (group B) were older than those in group A. Although differences between SBP and DBP were not statistically significant, the pulse pressure (PP) and mean arterial pressure (MAP) were significantly higher in patients from group $\mathrm{B}$ (with LVH) as compared to those from group A.

There were no significant differences between the two groups regarding lipid profile (total cholesterol, HDL cholesterol, LDL cholesterol, and triglycerides), serum glucose, and renal function (creatinine, urea). Albumin levels were significantly lower in patients in group B than in group A. Patients 
TABLE 2: Clinical, laboratory, and echocardiographic data of patients in the two groups.

\begin{tabular}{|c|c|c|c|}
\hline & Group A (HBP without LVH) & Group B (HBP with LVH) & $p$ \\
\hline Number & 38 & 49 & \\
\hline Age & $59.67 \pm 14.47$ & $65.17 \pm 8.501$ & .024 \\
\hline Men & $10(26.3 \%)$ & $16(32.6 \%)$ & .006 \\
\hline Women & $28(73.7 \%)$ & $33(67.4 \%)$ & .014 \\
\hline SBP (mmHg) & $151.00 \pm 26.91$ & $146.63 \pm 24.61$ & .796 \\
\hline DBP (mmHg) & $88.96 \pm 11.70$ & $95.55 \pm 77.61$ & .413 \\
\hline $\mathrm{PP}$ & $60.92 \pm 11.73$ & $62.04 \pm 18.37$ & .002 \\
\hline MAP & $106.16 \pm 13.11$ & $119.97 \pm 18.61$ & .001 \\
\hline BMI $\left(\mathrm{kg} / \mathrm{m}^{2}\right)$ & $30.49 \pm 5.19$ & $30.58 \pm 5.43$ & .03 \\
\hline Uric acid (mg/dL) & $6.57 \pm 1.76$ & $5.19 \pm 2.06$ & .079 \\
\hline Creatinine (mg/dL) & $0.88 \pm 0.49$ & $0.85 \pm 0.28$ & .246 \\
\hline Urea (mg/dL) & $31.02 \pm 21.91$ & $31.07 \pm 20.31$ & .790 \\
\hline Serum albumin (g/dL) & $3.5 \pm 0.2$ & $3.9 \pm 1.2$ & .000 \\
\hline Serum glucose (mg/dL) & $107.83 \pm 23.67$ & $107.97 \pm 35.40$ & .338 \\
\hline Total cholesterol (mg/dL) & $187.52 \pm 58.84$ & $201.79 \pm 57.34$ & .862 \\
\hline HDL cholesterol (mg/dL) & $47.05 \pm 8.94$ & $48.59 \pm 13.46$ & .577 \\
\hline LDL cholesterol (mg/dL) & $115.34 \pm 38.83$ & $117.38 \pm 42.91$ & .522 \\
\hline Triglycerides (mg/dL) & $128.73 \pm 89.81$ & $135.67 \pm 68.92$ & .070 \\
\hline $\mathrm{Na}(\mathrm{mEq} / \mathrm{L})$ & $138.58 \pm 2.81$ & $139.32 \pm 8.24$ & .476 \\
\hline $\mathrm{K}(\mathrm{mEq} / \mathrm{L})$ & $4.28 \pm 0.48$ & $4.38 \pm 0.53$ & .850 \\
\hline $\mathrm{ST} 2(\mathrm{ng} / \mathrm{mL})$ & $29.91 \pm 24.12$ & $36.03 \pm 29.91$ & .003 \\
\hline Ascending aorta (mm) & $26.46 \pm 8.97$ & $31.46 \pm 5.38$ & .002 \\
\hline Left atrium size (mm) & $31.13 \pm 8.98$ & $34.81 \pm 7.55$ & .056 \\
\hline Left atrium area $(\mathrm{mm})$ & $5.79 \pm 9.55$ & $9.74 \pm 10.63$ & .003 \\
\hline Left atrium area index $(\mathrm{mm})$ & $21.32 \pm 8.56$ & $28.47 \pm 7.21$ & .001 \\
\hline End-systolic interventricular septum (mm) & $9.46 \pm 5.37$ & $13.92 \pm 5.78$ & .002 \\
\hline End-diastolic interventricular septum (mm) & $9.71 \pm 1.53$ & $13.01 \pm 1.56$ & .000 \\
\hline End-systolic LV posterior wall (mm) & $10.78 \pm 4.77$ & $13.88 \pm 5.13$ & .012 \\
\hline End-diastolic LV posterior wall (mm) & $10.01 \pm 1.43$ & $12.11 \pm 2.32$ & .000 \\
\hline End-systolic LV size (mm) & $24.77 \pm 14.07$ & $28.30 \pm 11.17$ & .224 \\
\hline End-diastolic LV size (mm) & $42.52 \pm 6.11$ & $44.57 \pm 8.95$ & .306 \\
\hline Right ventricle size (mm) & $26.35 \pm 4.28$ & $26.28 \pm 9.11$ & .074 \\
\hline Stroke volume $(\mathrm{mL})$ & $13.92 \pm 22.96$ & $37.37 \pm 34.70$ & .001 \\
\hline LV mass $\left(\mathrm{g} / \mathrm{m}^{2}\right)$ & $145.4 \pm 62.2$ & $262.9 \pm 83.6$ & .000 \\
\hline LV mass index & $44.95 \pm 85.30$ & $164.15 \pm 128.93$ & .000 \\
\hline IVRT (ms) & $127.96 \pm 72.47$ & $98.38 \pm 49.03$ & .009 \\
\hline EDT (ms) & $211.72 \pm 120.06$ & $218.64 \pm 71.65$ & .005 \\
\hline
\end{tabular}

HBP: hypertension; LVH: left ventricular hypertrophy; SBP: systolic blood pressure; DBP: diastolic blood pressure; PP: pulse pressure; MAP: mean arterial pressure; BMI: body mass index; LV: left ventricle; IVRT: isovolumetric relaxation time; EDT: mitral $E$ wave deceleration time.

in group B had significantly higher LA area, ascending aorta diameter, interventricular septum, and LV posterior wall thickness than those in group A. Additionally, patients in group B had lower IVRT and higher $E$ wave DT than patients without LVH. LV ejection fraction was normal $(>50 \%)$ in all patients in the study groups.

The sST2 levels were significantly higher in patients from group $\mathrm{B}$ (with $\mathrm{LVH}$ ) than in those from group A (without HVS) $(36.03 \mathrm{ng} / \mathrm{mL}$ versus $29.91 \mathrm{ng} / \mathrm{mL}$, $p=0.003)$. sST2 was negatively correlated with the degree of $\operatorname{LVH}(r=-0.45, p=0.034)$.
ROC curves for sST2 showed that a cutoff value of $14.04 \mathrm{ng} / \mathrm{mL}$ had an $82.1 \%$ sensitivity and $53.8 \%$ specificity (AUC $=0.771)$ to discriminate between patients with and without LVH (Figure 1(a)). The sensitivity and specificity of SST2 levels of $>24.18 \mathrm{ng} / \mathrm{mL}$ for the discrimination between grade 2 diastolic dysfunction and normal diastolic function in hypertensive patients were $94.4 \%$ and $69.1 \%$, respectively (AUC $=0.721, p=0.004$, Figure $1(\mathrm{~b})$ ).

To further assess the performance of sST2 as a predictor of the presence of LVH in patients with arterial hypertension, we compared it with the performance of proBNP, which is 


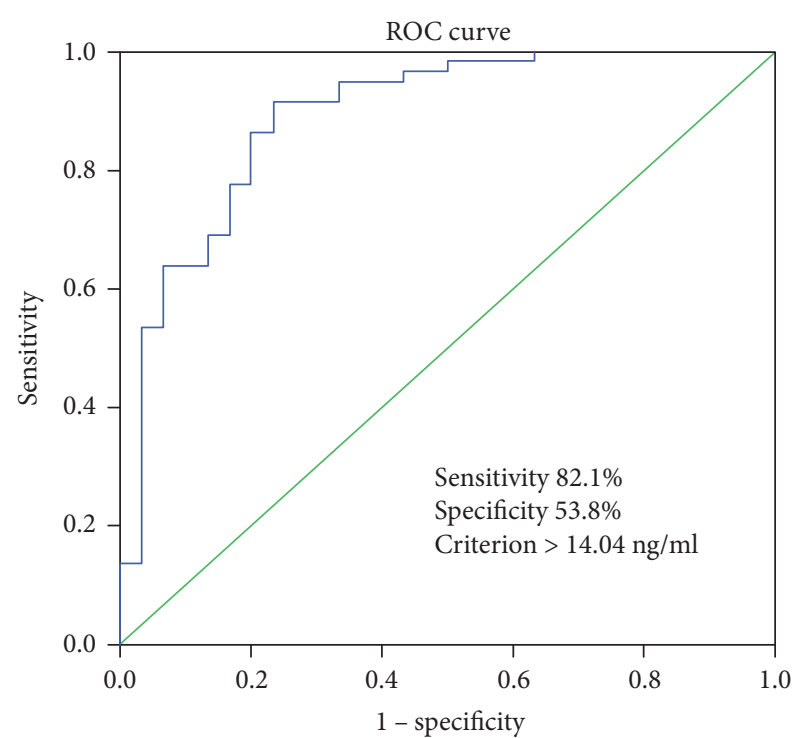

(a)

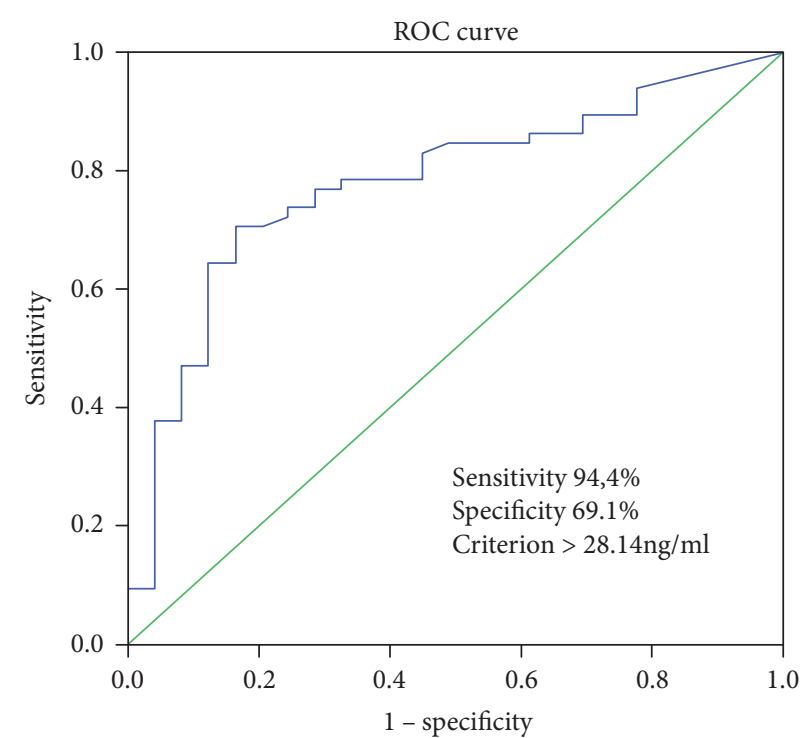

(b)

FIGURE 1: (a) ROC analysis showing the sST2 sensitivity and specificity for predicting the presence of LVH in patients with arterial hypertension. (b) ROC analysis showing ST2 sensitivity and specificity for the presence of grade 2 diastolic dysfunction in hypertensive patients.

considered a reference (Figure 2). The AUC values for sST2 and proBNP were 0.732 (95\% CI: $0.613-0.850)$ and 0.669 (95\% CI: 0.524-0.814), respectively.

Correlation analysis with log-transformed sST2 as a dependent variable showed that this parameter was significantly and positively correlated with ascending aorta diameter, LV mass, end-systolic LV size, end-diastolic LV size, enddiastolic LV posterior wall, and end-diastolic interventricular septum. Regarding the diastolic function parameters, we found that SST2 was significantly and positively correlated with the E/A ratio and E/e'm and negatively correlated with ejection time (Table 3). No correlation was observed between sST2 and ejection fraction and left atrium area. By linear regression analysis, we found that $E / A, E / \mathrm{e}^{\prime} \mathrm{m}$, and LVET predicted $35.6 \%$ of the variability of the sST2 levels [ $F$ $(3.82)=3.940, p=0.011)]$. We also noticed that ST2 levels increased with the additional CV risk, $5.3 \%$ of ST2 level variability being explained by a 1 -unit variation in this risk ( $\beta=8.3, p=0.020$, 95\% CI 1.36-15.25) (data not shown).

By regression analysis, we showed that $33 \%$ of the variance in LV mass can be explained by a $10 \mathrm{mmHg}$ variance of PP $(\beta=0.2, p=0.049,95 \%$ CI $0.008-0.34)$.

sST2 levels increased proportionally with the presence and worsening of the diastolic dysfunction (Table 4). The highest ST2 levels were observed in stage 2 diastolic dysfunction compared to no diastolic dysfunction. In patients with diastolic dysfunction, the differences in serum ST2 levels between stage 2 versus none and stage 1 versus none, although present, did not reach statistical significance.

\section{Discussion}

Our study shows that in hypertensive patients, serum ST2 levels increase in parallel with LV mass and severity of

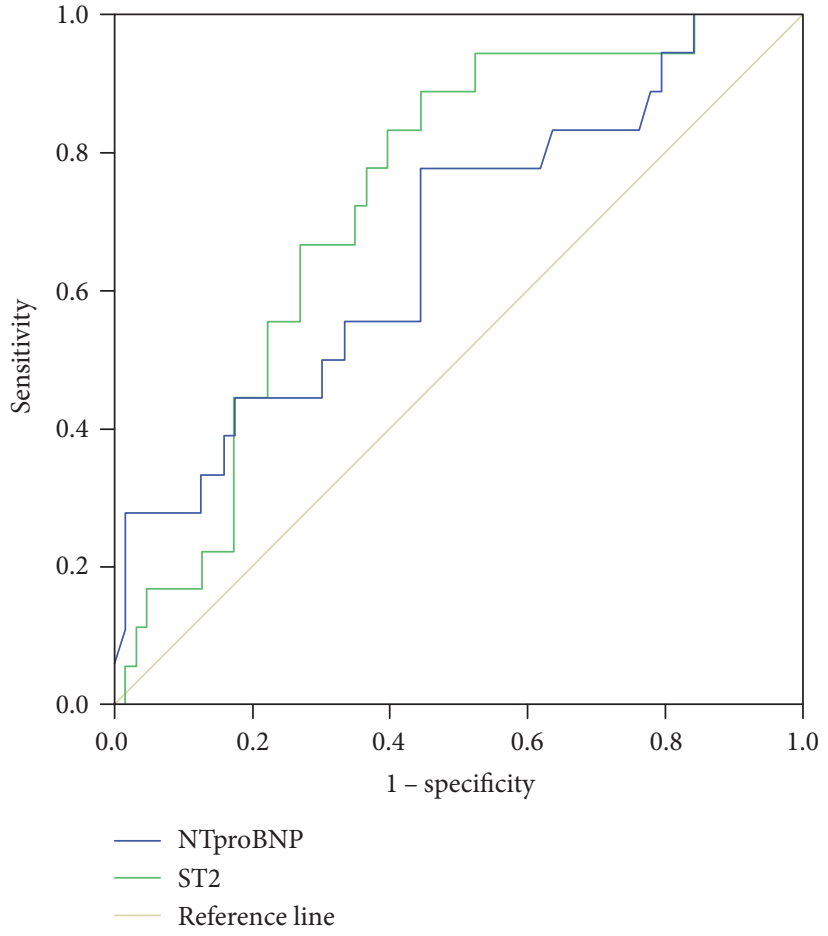

FIGURE 2: ROC analysis for the comparison between sST2 and proBNP as predictors of the presence of $\mathrm{LVH}$ in patients with arterial hypertension.

myocardial dysfunction. Serum ST2 levels increased linearly with the degree of LVH (assessed with end-diastolic IV septum, end-diastolic LW posterior wall, and LV mass). ST2 has proven to be a useful biomarker for diagnosing $\mathrm{LVH}$, with an $82.1 \%$ sensitivity and $53.8 \%$ specificity for values $>14.04 \mathrm{ng} / \mathrm{mL}$. Ojji et al. found similar results in 
TABLE 3: Correlation of clinical/echocardiographic parameters with log-transformed ST2 and diastolic dysfunction.

\begin{tabular}{|c|c|c|}
\hline Log-transformed ST2 & $\begin{array}{l}\text { Correlation } \\
\text { coefficient }\end{array}$ & $p$ \\
\hline BMI & 0.21 & .056 \\
\hline SBP & 0.127 & .237 \\
\hline DBP & 0.03 & .981 \\
\hline $\mathrm{PP}$ & 0.178 & .097 \\
\hline MAP & 0.071 & .509 \\
\hline CVAR & 0.253 & .023 \\
\hline Ascending aorta & 0.212 & .048 \\
\hline Left atrium area & 0.16 & .21 \\
\hline $\begin{array}{l}\text { End-diastolic interventricular } \\
\text { septum }\end{array}$ & 0.51 & .000 \\
\hline End-diastolic LV posterior wall & 0.40 & .03 \\
\hline End-diastolic LV size & 0.28 & .02 \\
\hline End-systolic LV size & 0.35 & .01 \\
\hline LV mass index & 0.44 & .000 \\
\hline Ejection fraction & 0.119 & .268 \\
\hline$E / A$ & 0.22 & .03 \\
\hline E/e'm & 0.215 & .02 \\
\hline Ejection time & -0.259 & .015 \\
\hline Diastolic dysfunction & $\begin{array}{l}\text { Correlation } \\
\text { coefficient }\end{array}$ & $p$ \\
\hline Ascending aorta & 0.212 & .048 \\
\hline Left atrium area & 0.16 & .21 \\
\hline $\begin{array}{l}\text { End-diastolic interventricular } \\
\text { septum }\end{array}$ & 0.51 & .000 \\
\hline End-diastolic LV posterior wall & 0.40 & .03 \\
\hline End-diastolic LV size & 0.28 & .02 \\
\hline End-systolic LV size & 0.35 & .01 \\
\hline LV mass index & 0.44 & .000 \\
\hline Peak E/A ratio & 0.22 & .03 \\
\hline Myocardial performance index & 0.245 & .012 \\
\hline ST2 & 0.273 & .006 \\
\hline E/e'm & 0.547 & .000 \\
\hline Ejection time & 0.235 & .015 \\
\hline
\end{tabular}

HBP: hypertension; LVH: left ventricular hypertrophy; SBP: systolic blood pressure; DBP: diastolic blood pressure; PP: pulse pressure; MAP: mean arterial pressure; BMI: body mass index; LV: left ventricle; CVAR: cardiovascular additional risk; $A$ : late diastolic mitral peak filling velocity; $E$ : early diastolic mitral peak filling velocity; e'm: peak early diastolic mitral annulus velocity by tissue Doppler.

a cohort of hypertensive patients [20]. In 210 hypertensive patients, the authors showed that plasma ST2 levels were significantly higher in those with LVH compared to those without LVH $(134.7 \mathrm{ng} / \mathrm{mL}$ versus $23.0 \mathrm{ng} / \mathrm{mL})$ [20]. The relationship between serum ST2 levels and hypertrophic myocardium was previously shown by Sanada et al. [14] who showed that the IL-33/ST2L system is biomechanically activated and regulates cardiomyocyte hypertrophy and cardiac fibrosis.
In our study, we found serum ST2 levels had a significant and positive correlation with echocardiographic parameters of impaired relaxation (E/A ratio) and LV filling pressure (E/e'm ratio; correlation coefficients of 0.22 and 0.215 , respectively; $p<0.05$ for both) and had a good performance in predicting the presence of LV diastolic dysfunction in hypertensive patients. These results are in line with the ones previously reported by Ojji et al. in hypertensive patients which showed that sST2 levels were correlated with echocardiographic parameters of diastolic function - transmitral $E / A$ ratio (Pearson correlation coefficient of 0.43) [20]. Furthermore, we showed that serum ST2 levels have the ability to discriminate between grade 2 diastolic dysfunction and normal diastolic performance, with a sensitivity of $94.4 \%$ and a specificity of $69.1 \%$ at levels higher than $24.18 \mathrm{ng} / \mathrm{mL}$. To the best of our knowledge, these are the first published data regarding the ability of ST2 to quantify diastolic dysfunction severity in hypertensive patients and to provide a cutoff value for ST2. The recognition of LV diastolic dysfunction, regardless of systolic performance, as a distinct entity and as a negative prognostic marker has prompted a considerable amount of research for its early identification. The current state-of-the-art diagnosis involves echocardiography and is limited by technical factors (image quality, Doppler flow patterns, and availability of tissue Doppler imaging) and patient characteristics such as overweight and chest hyperinflation $[18,19]$. Thus, the evaluation of ST2 may add important information to support the diagnosis of LV diastolic dysfunction. It has been previously observed that serum ST2 levels increase in parallel with the myocardial mass, and thus, it was initially hypothesized that high levels of ST2 are produced by the myocardium in response to volume and pressure overload [21]. In a study on ST2 and hemodynamic parameters in pressure overload hypertrophy (patients with aortic stenosis), Bartunek et al. [22] showed that diastolic load indeed modulates the production of ST2. Surprisingly, increased serum ST2 levels were produced not by the hypertrophied myocardium but rather by the vascular endothelial cells in response to the diastolic load [22].

We have also studied the correlation of ST2 levels with $\mathrm{LV}$ mass and its ability to predict the presence of LVH in our sample of hypertensive patients. ST2 was correlated with LV mass in our sample of hypertensive patients, displaying a correlation coefficient of $0.44(p<0.0001)$. We showed that ST2 levels of $14.04 \mathrm{ng} / \mathrm{mL}$ or above identify patients with LVH with a sensitivity of $82.1 \%$ and a specificity of $53.8 \%$. As compared to that of proBNP, the performance of these ST2 levels were slightly improved, as shown by the AUC values higher for ST2 than for proBNP (0.732 versus 0.669$)$. Our results confirm the previous observations reporting a higher performance of ST2 than the one of proBNP in predicting the presence of LVH in hypertensive patients [20].

Although in hypertension, LVH is an adaptive response to increased LV wall stress, the progression of myocardial hypertrophy is regulated by several hemodynamic and humoral factors. In our study, a $1 \mathrm{mmHg}$ increase in pulse pressure-as a consequence of arterial stiffness induced by hypertension, age, smoking, and dyslipidemia [23] —-was responsible for $3.3 \%$ of the LV mass variability. Pulse 
TABLE 4: Serum sST2 levels of patients according to the presence of diastolic dysfunction and cardiovascular risk.

\begin{tabular}{lccc}
\hline & & ST2 $(\mathrm{ng} / \mathrm{mL})$ & $p$ \\
\hline \multirow{4}{*}{ E/e'm } & $\leq 8$ & $30.67 \pm 22.18$ & $0.03^{\mathrm{a}}$ \\
& $9-12$ & $33.34 \pm 27.56$ & $0.002^{\mathrm{b}}$ \\
& $\geq 13$ & $42.16 \pm 39.51$ & $.000^{\mathrm{c}}$ \\
\hline \multirow{3}{*}{ Diastolic dysfunction } & Absent & $27.06 \pm 12.97$ & $.048^{\mathrm{d}}$ \\
& Grade 1/mild & $30.67 \pm 21.06$ & $.016^{\mathrm{e}}$ \\
& Grade 2/moderate & $43.54 \pm 17.49$ & $.008^{\mathrm{f}}$ \\
\hline \multirow{2}{*}{ Diabetes } & Absent & $32.76 \pm 20.61$ & $.002^{\mathrm{g}}$ \\
& Present & $39.16 \pm 40.98$ & \\
\hline \multirow{3}{*}{ Additional CV risk } & Moderate & $23.66 \pm 15.73$ & $.020^{\mathrm{h}}$ \\
& High & $31.58 \pm 20.96$ & $.04^{\mathrm{i}}$ \\
& Very high & $40.16 \pm 28.03$ & $.026^{\mathrm{j}}$ \\
\hline
\end{tabular}

${ }^{\mathrm{a}} E / \mathrm{e}^{\prime} \mathrm{m} \leq 8$ versus $E / \mathrm{e}^{\prime} \mathrm{m}=9-12 ;{ }^{\mathrm{b}} E / \mathrm{e}^{\prime} \mathrm{m}=9-12$ versus $E / \mathrm{e}^{\prime} \mathrm{m} \geq 13 ;{ }^{\mathrm{c}} E / \mathrm{e}^{\prime} \mathrm{m} \leq 8$ versus $E / e^{\prime} m \geq 13$; ${ }^{\mathrm{d}}$ absent versus grade 1 ; ${ }^{\mathrm{e}}$ grade 1 versus grade 2 ; ${ }^{\mathrm{f}}$ absent versus grade 2 ; ${ }^{\mathrm{g}}$ absent versus present; ${ }^{\mathrm{h}}$ moderate versus high; ${ }^{\mathrm{i}}$ high versus very high; ${ }^{\mathrm{j}}$ moderate versus very high; CV: cardiovascular.

pressure was previously proven to be an independent prognostic factor for $\mathrm{CV}$ events in patients with hypertension [23], cost-effective, and easily quantifiable in the examination room.

We found that serum albumin levels were significantly lower in hypertensive patients with LVH and had a negative correlation with echocardiographic parameters for LV remodeling (LVH, left atrium size, and end-diastolic IV septum). The study by Ahbap et al. [24] found that serum albumin was lower in nondipper hypertensive patients compared to dippers, even for similar levels of proteinuria. Therefore, they postulated that serum albumin rather than urinary albumin excretion is an independent predictor for nocturnal dips in SBP and DBP (nocturnal SBP and DBP variability). 24 hour BP variability, daylight BP variability, and BP duration and severity are significant hemodynamic factors involved in LVH pathophysiology $[25,26]$.

Given all things were considered, cutoff serum ST2 values to identify LVH or quantify the level of LV diastolic dysfunction could be the milestones for the progression towards HF in hypertensive patients and identify patients with high risk of unfavorable outcome. Of course, these cutoff values would need to be validated on large population trials.

5.1. Limitations. Our study has several limitations. First, it enrolled a rather small group of patients; that is why we believe the relationship between ST2 and diastolic dysfunction should be evaluated in larger groups of patients with hypertension and also other CV diseases that decrease mainly the diastolic performance. The results we obtained could provide additional working hypotheses for future work. Secondly, we cannot exclude the presence of a subclinical myocardial ischemia that was unidentifiable by collecting medical history and ECG, although it is known that only myocardial remodeling secondary to acute ischemia is associated with increased serum ST2 levels [27, 28]. Thirdly, onethird of patients had diabetes-a condition characterized by a significant inflammatory status and associated with high serum ST2 levels (even in the absence of LVH) [29] —and this might have influenced our results. Additionally, we did not assess intra- and interday variability of ST2 levels measured by the assay used. To overcome the potential intraday variability, we collected all samples in the morning in fasting conditions. We could not identify any reports in the literature on the biological variability of the ST2 levels measured by the assay we used. However, assessments using a Presage ST2 assay showed that ST2 has an intraindividual coefficient of variation over a moderate time interval of $11 \%$, lower as compared to the one of proBNP and is not influenced by the fasting or nonfasting status $[30,31]$.

Although with these limitations, our study offers valuable data on a real-life cohort of hypertensive patients.

\section{Conflicts of Interest}

The authors have no conflict of interest to declare.

\section{Authors' Contributions}

Anca Daniela Farcaş, Florin Petru Anton, and Mirela Anca Stoia conceived the study design. Anca Daniela Farcaş and Florin Petru Anton made the acquisition, analysis, or interpretation of the data of the work and drafted the manuscript. Florin Petru Anton made the statistical analysis of the databases. Florin Petru Anton and Cerasela Mihaela Goidescu analyzed and helped with the interpretation of the data of the work. Iulia Laura Gavrilă collected the data and built the database. Luminița Animarie Vida-Simiti revised the manuscript critically for important intellectual content. All authors read and approved the final manuscript.

\section{Acknowledgments}

The authors are grateful to their laboratory colleagues for their everyday work in assessing the biological sample. They want to thank Mihai Lapadat for his great support in editing the manuscript.

\section{References}

[1] D. Levy, R. J. Garrison, D. D. Savage, W. B. Kannel, and W. P. Castelli, "Prognostic implications of echocardiographically determined left ventricular mass in the Framingham Heart Study," The New England Journal of Medicine, vol. 322, no. 22, pp. 1561-1566, 1990.

[2] G. de Simone, J. S. Gottdiener, M. Chinali, and M. S. Maurer, "Left ventricular mass predicts heart failure not related to previous myocardial infarction: the Cardiovascular Health Study," European Heart Journal, vol. 29, no. 6, pp. 741-747, 2008.

[3] A. Ganau, R. B. Devereux, M. J. Roman et al., "Patterns of left ventricular hypertrophy and geometric remodeling in essential hypertension," Journal of the American College of Cardiology, vol. 19, no. 7, pp. 1550-1558, 1992.

[4] G. de Simone, D. W. Kitzman, M. Chinali et al., "Left ventricular concentric geometry is associated with impaired 
relaxation in hypertension: the HyperGEN study," European Heart Journal, vol. 26, no. 10, pp. 1039-1045, 2005.

[5] N. S. Chahal, T. K. Lim, P. Jain, J. C. Chambers, J. S. Kooner, and R. Senior, "New insights into the relationship of left ventricular geometry and left ventricular mass with cardiac function: a population study of hypertensive subjects," European Heart Journal, vol. 31, no. 5, pp. 588-594, 2010.

[6] T. H. Marwick, T. C. Gillebert, G. Aurigemma et al., "Recommendations on the use of echocardiography in adult hypertension: a report from the European Association of Cardiovascular Imaging (EACVI) and the American Society of Echocardiography (ASE) dagger," European Heart Journal. Cardiovascular Imaging, vol. 16, no. 6, pp. 577-605, 2015.

[7] M. Santos and A. M. Shah, "Alterations in cardiac structure and function in hypertension," Current Hypertension Reports, vol. 16, no. 5, p. 428, 2014.

[8] S. Nistri, P. Ballo, D. Mele et al., "Effect of echocardiographic grading of left ventricular diastolic dysfunction by different classifications in primary care," The American Journal of Cardiology, vol. 116, no. 7, pp. 1144-1152, 2015.

[9] M. S. Sabatine, D. A. Morrow, L. J. Higgins et al., "Complementary roles for biomarkers of biomechanical strain ST2 and N-terminal prohormone B-type natriuretic peptide in patients with ST-elevation myocardial infarction," Circulation, vol. 117, no. 15, pp. 1936-1944, 2008.

[10] J. L. Januzzi Jr., W. F. Peacock, A. S. Maisel et al., "Measurement of the interleukin family member ST2 in patients with acute dyspnea: results from the PRIDE (Pro-Brain Natriuretic Peptide Investigation of Dyspnea in the Emergency Department) study," Journal of the American College of Cardiology, vol. 50, no. 7, pp. 607-613, 2007.

[11] N. Iqbal, B. Wentworth, R. Choudhary et al., "Cardiac biomarkers: new tools for heart failure management," Cardiovascular Diagnosis and Therapy, vol. 2, no. 2, pp. 147-164, 2012.

[12] T. J. Wang, K. C. Wollert, M. G. Larson et al., "Prognostic utility of novel biomarkers of cardiovascular stress: the Framingham Heart Study," Circulation, vol. 126, no. 13, pp. 1596-1604, 2012.

[13] E. E. Coglianese, M. G. Larson, R. S. Vasan et al., "Distribution and clinical correlates of the interleukin receptor family member soluble ST2 in the Framingham Heart Study," Clinical Chemistry, vol. 58, no. 12, pp. 1673-1681, 2012.

[14] S. Sanada, D. Hakuno, L. J. Higgins, E. R. Schreiter, A. N. McKenzie, and R. T. Lee, "IL-33 and ST2 comprise a critical biomechanically induced and cardioprotective signaling system," The Journal of Clinical Investigation, vol. 117, no. 6, pp. 1538-1549, 2007.

[15] A. J. Coyle, C. Lloyd, J. Tian et al., "Crucial role of the interleukin 1 receptor family member T1/ST2 in T helper cell type 2mediated lung mucosal immune responses," The Journal of Experimental Medicine, vol. 190, no. 7, pp. 895-902, 1999.

[16] G. Mancia, R. Fagard, K. Narkiewicz et al., "2013 ESH/ESC guidelines for the management of arterial hypertension: the task force for the management of arterial hypertension of the European Society of Hypertension (ESH) and of the European Society of Cardiology (ESC)," Journal of Hypertension, vol. 31, no. 7, pp. 1281-1357, 2013.

[17] Recommendations for cardiac chamber quantification by echocardiography in adults: an update from the American Society of Echocardiography and the European Association of Cardiovascular Imaging," European Heart Journal. Cardiovascular Imaging, vol. 17, no. 4, p. 412, 2016.
[18] S. F. Nagueh, O. A. Smiseth, C. P. Appleton et al., "Recommendations for the evaluation of left ventricular diastolic function by echocardiography: an update from the American Society of Echocardiography and the European Association of Cardiovascular Imaging," European Heart Journal. Cardiovascular Imaging, vol. 17, no. 12, pp. 1321-1360, 2016.

[19] R. M. Lang, L. P. Badano, V. Mor-Avi et al., "Recommendations for cardiac chamber quantification by echocardiography in adults: an update from the American Society of Echocardiography and the European Association of Cardiovascular Imaging," European Heart Journal. Cardiovascular Imaging, vol. 16, no. 3, pp. 233-270, 2015.

[20] D. B. Ojji, L. H. Opie, S. Lecour, L. Lacerda, O. M. Adeyemi, and K. Sliwa, "The effect of left ventricular remodelling on soluble ST2 in a cohort of hypertensive subjects," Journal of Human Hypertension, vol. 28, no. 7, pp. 432-437, 2014.

[21] M. Vanderheyden, M. Goethals, S. Verstreken et al., "Wall stress modulates brain natriuretic peptide production in pressure overload cardiomyopathy," Journal of the American College of Cardiology, vol. 44, no. 12, pp. 2349-2354, 2004

[22] J. Bartunek, L. Delrue, F. Van Durme et al., "Nonmyocardial production of ST2 protein in human hypertrophy and failure is related to diastolic load," Journal of the American College of Cardiology, vol. 52, no. 25, pp. 2166-2174, 2008.

[23] C. F. Wu, P. Y. Liu, T. J. Wu, Y. Hung, S. P. Yang, and G. M. Lin, "Therapeutic modification of arterial stiffness: an update and comprehensive review," World Journal of Cardiology, vol. 7, no. 11, pp. 742-753, 2015.

[24] E. Ahbap, T. Sakaci, E. Kara et al., "The relationship between serum albumin levels and 24-h ambulatory blood pressure monitoring recordings in non-diabetic essential hypertensive patients," Clinics (São Paulo, Brazil), vol. 71, no. 5, pp. 257263, 2016.

[25] G. Parati, G. Pomidossi, F. Albini, D. Malaspina, and G. Mancia, "Relationship of 24-hour blood pressure mean and variability to severity of target-organ damage in hypertension," Journal of Hypertension, vol. 5, no. 1, pp. 93-98, 1987.

[26] R. B. Devereux and T. G. Pickering, "Relationship between the level, pattern and variability of ambulatory blood pressure and target organ damage in hypertension," Journal of Hypertension. Supplement, vol. 9, no. 8, pp. S34-S38, 1991.

[27] S. Demyanets, W. S. Speidl, I. Tentzeris et al., "Soluble ST2 and interleukin-33 levels in coronary artery disease: relation to disease activity and adverse outcome," PLoS One, vol. 9, no. 4, article e95055, 2014.

[28] J. Yayan, "Emerging families of biomarkers for coronary artery disease: inflammatory mediators," Vascular Health and Risk Management, vol. 9, pp. 435-456, 2013.

[29] E. Fousteris, A. Melidonis, G. Panoutsopoulos et al., "Toll/ interleukin-1 receptor member ST2 exhibits higher soluble levels in type 2 diabetes, especially when accompanied with left ventricular diastolic dysfunction," Cardiovascular Diabetology, vol. 10, p. 101, 2011.

[30] B. Dieplinger, M. Egger, A. Gegenhuber, M. Haltmayer, and T. Mueller, "Analytical and clinical evaluation of a novel high-sensitivity assay for measurement of soluble ST2 in human plasma - The presage ${ }^{\mathrm{TM}}$ ST2 assay," Clinica Chimica Acta, vol. 451, no. part B, pp. 310-315, 2015.

[31] A. H. Wu, F. Wians, and A. Jaffe, "Biological variation of galectin-3 and soluble ST2 for chronic heart failure: implication on interpretation of test results," American Heart Journal, vol. 165, no. 6, pp. 995-999, 2013. 


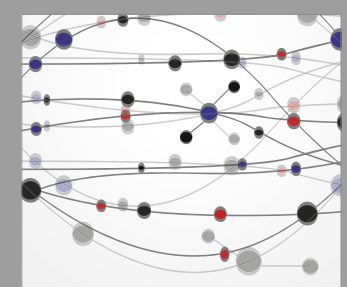

The Scientific World Journal
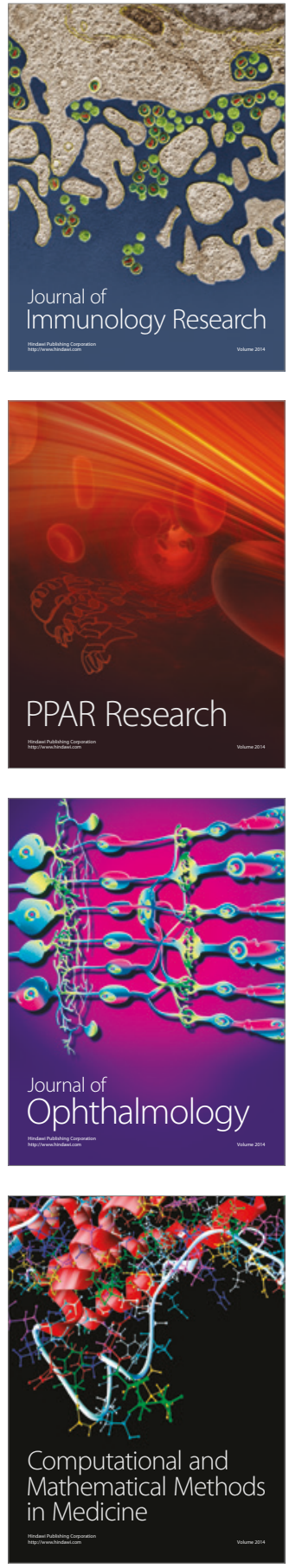

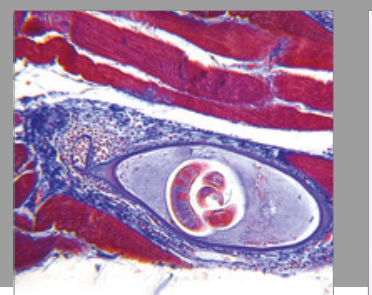

Gastroenterology Research and Practice
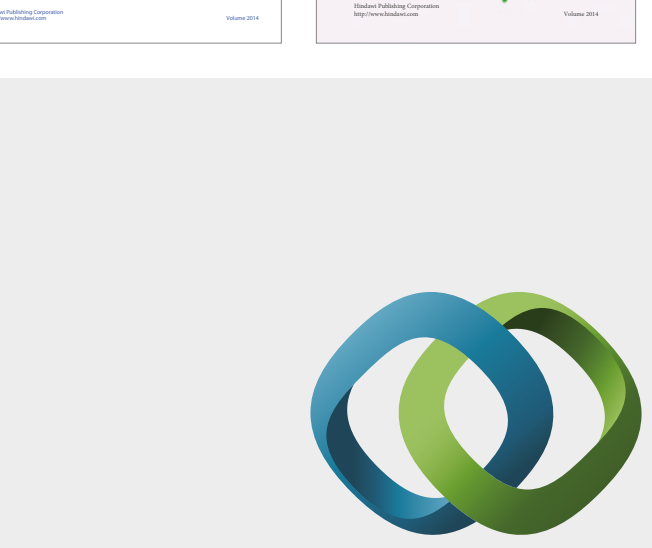

\section{Hindawi}

Submit your manuscripts at

https://www.hindawi.com
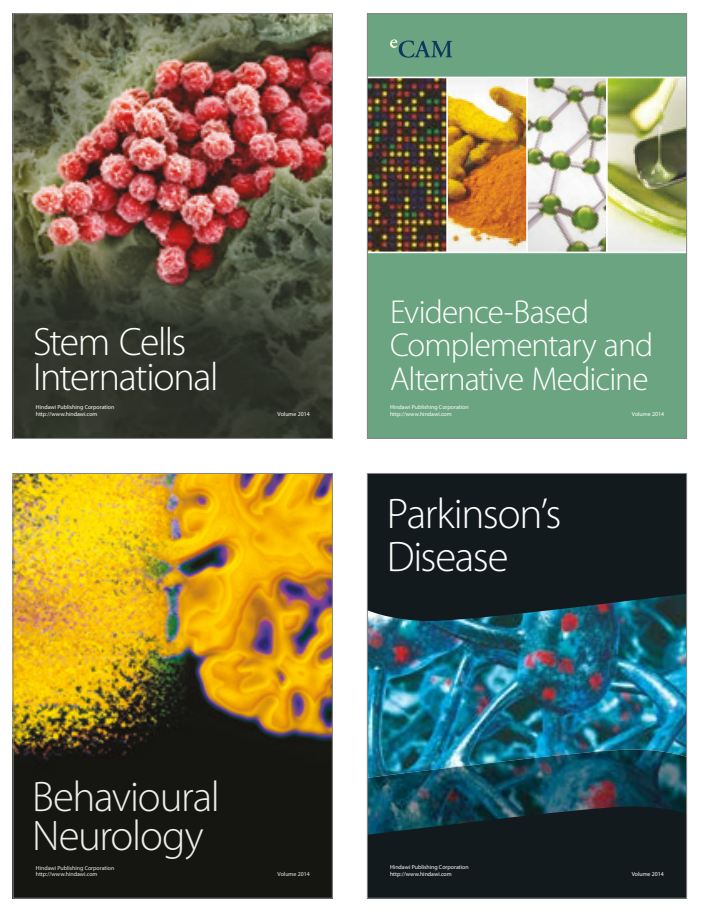
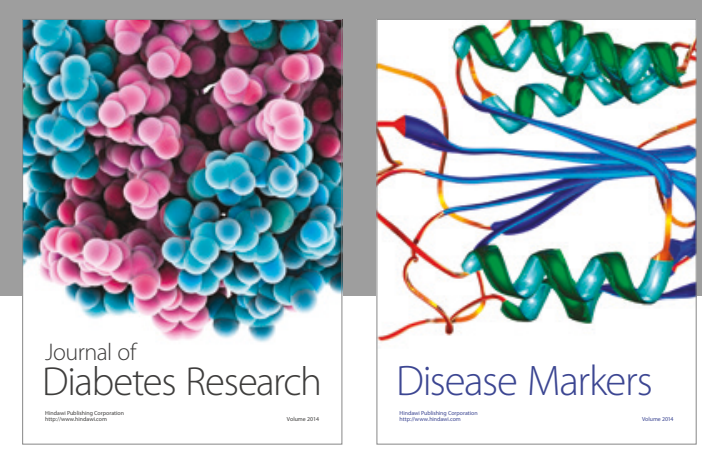

Disease Markers
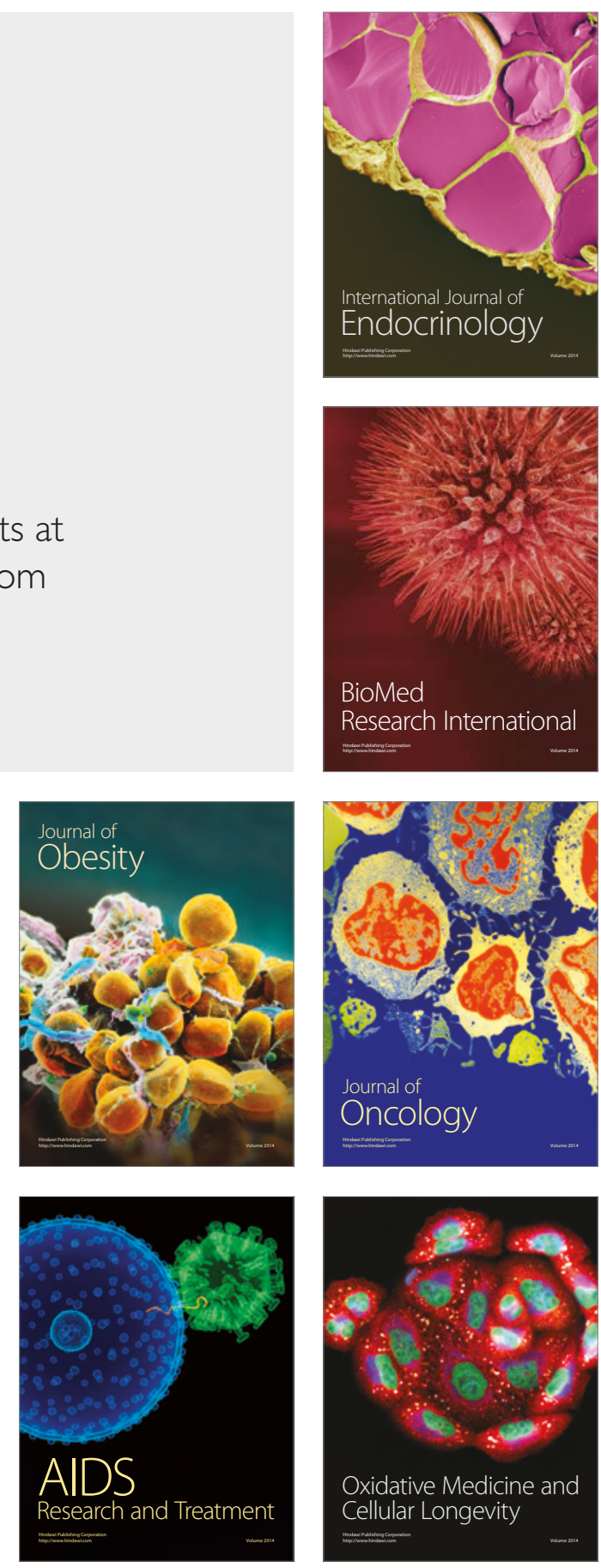